\title{
Diagnostic Value of Volume-Based Fluorine-18- Fluorodeoxyglucose PET/CT Parameters for Characterizing Thyroid Incidentaloma
}

Huazheng Shi, MD ${ }^{1,2,3 *}$, Zuguo Yuan, MD ${ }^{4 *}$, Zheng Yuan, MD ${ }^{1,2}$, Chunshan Yang, MD ${ }^{1,2}$, Jian Zhang, MD ${ }^{1,2}$ Yi Shou, $M D^{1,2}$, Wenrui Zhang, $M D^{1,2}$, Zhaofu Ping, $M D^{1,2}$, Xin Gao, MD ${ }^{1,2}$, Shiyuan Liu, MD, PhD ${ }^{3}$

${ }^{1}$ Shanghai Universal Medical Imaging Diagnostic Center, Shanghai 200233, China; ${ }^{2}$ Department of Radiology, PET/CT Center, Shanghai 85 Hospital, Shanghai 200052, China; ${ }^{3}$ Department of Radiology, affiliated Changzheng Hospital, The Second Military Medical University, Shanghai 200003, China; 'Department of Chemoradiotherapy, Yinzhou People's Hospital, Ningbo 315100, China

Objective: To assess clinical value of fluorine-18-fluorodeoxyglucose positron emission tomography/computed tomography (PET/CT) for differentiation of malignant from benign focal thyroid incidentaloma.

Materials and Methods: This retrospective study included 99 patients with focal thyroid incidentaloma of 5216 non-thyroid cancer patients that had undergone PET/CT. PET/CT semi-quantitative parameters, volume-based functional parameters, metabolic tumor volume (MTV), and total lesion glycolysis (TLG) of thyroid incidentaloma were assessed. Receiver-operating characteristic (ROC) analysis was conducted and areas under the curve (AUC) were compared by Hanley and McNeil test to evaluate usefulness of maximum standardized uptake value (SUVmax), MTV and TLG, as markers for differentiating malignant from benign thyroid incidentalomas.

Results: Of 99 thyroid incidentalomas, 64 (64.6\%) were malignant and 35 (35.4\%) were benign. Malignant thyroid incidentalomas were larger (1.8 cm vs. $1.3 \mathrm{~cm}, p=0.006)$, and had higher SUVmax (11.3 vs. 4.8, $p<0.001)$, MTV (all $p<$ 0.001 ) and TLG (all $p<0.001$ ) than benign. TLG 4.0 had the highest performance for differentiation of malignant from benign thyroid incidentaloma in all semi-quantitative parameters with AUC 0.895 by ROC curve analysis. AUC (TLG 4.0) was significantly larger than AUC (SUVmean), AUC (MTV 2.5), AUC (MTV 3.0), AUC (MTV 3.5), AUC (TLG 2.5), and AUC (TLG 3.0), respectively (all, $p<0.05)$. There was no statistical difference between AUC (TLG 4.0) and AUC (SUVmax) $(p>0.05)$. A threshold TLG 4.0 of 2.475 had $81.3 \%$ sensitivity and $94.3 \%$ specificity for identifying malignant thyroid incidentalomas.

Conclusion: Volume-based PET/CT parameters could potentially have clinical value in differential diagnosis of thyroid incidentaloma along with SUVmax.

Keywords: Fluorine-18-fluorodeoxyglucose; FDG PET/CT; Thyroid neoplasms; Metabolic tumor volume; Total lesion glycolysis; Thyroid incidentaloma

Received January 24, 2017; accepted after revision August 20, 2017. This study was partly supported by Shanghai Municipal of Health and Family Planning Commission (20164Y0014).

*These authors contributed equally to this work.

Corresponding author: Zheng Yuan, MD, Department of Radiology, PET/CT Center, Shanghai 85 Hospital, 1328 Hua Shan Road, Shanghai 200052, China.

- Tel: (8621) 81818186 - Fax: (8621) 81818186

- E-mail: yuanzheng0404@163.com

This is an Open Access article distributed under the terms of the Creative Commons Attribution Non-Commercial License (http://creativecommons.org/licenses/by-nc/4.0) which permits unrestricted non-commercial use, distribution, and reproduction in any medium, provided the original work is properly cited.

\section{INTRODUCTION}

Thyroid incidentalomas are defined as newly detected thyroid lesions on an imaging study for non-thyroid disease (1). As fluorine-18-fluorodeoxyglucose positron emission tomography/computed tomography $\left({ }^{18} \mathrm{~F}-\mathrm{FDG}-\mathrm{PET} / \mathrm{CT}\right)$ is widely used in diagnosis, tumor staging, and monitoring of treatment responses in various malignancies (2), incidence of thyroid ${ }^{18} \mathrm{~F}$-FDG incidentaloma is increasing $(3,4)$. Unlike diffuse thyroid uptake on FDG PET/CT considered more often benign (5-7), focal ${ }^{18} \mathrm{~F}-\mathrm{FDG}$ uptake of the thyroid on ${ }^{18} \mathrm{~F}-\mathrm{FDG}$ 
$\mathrm{PET} / \mathrm{CT}$ is more clinically significant because of its high risk for malignant disease (8-10).

Differentiation between malignant and benign thyroid nodules is essential for reducing unnecessary operations and for determining the patient's prognosis. However, it is difficult to distinguish benign from malignant nodules with conventional imaging techniques. Maximum standardized uptake value (SUVmax) cannot reflect actual tumor heterogeneity metabolic activity in the whole tumor, as it reflects one pixel value. Whether SUVmax can differentiate malignant from benign thyroid nodules is a highly debated issue $(9,11,12) .{ }^{18} \mathrm{~F}-\mathrm{FDG}$ metabolic tumor volume (MTV) and total lesion glycolysis (TLG), combining tumor volume and metabolic activity of the entire tumor, are semi-quantitative parameters for assessing metabolic activity of tumors determined by ${ }^{18} \mathrm{~F}-\mathrm{FDG}$ PET/CT images, that may overcome this potential issue. MTV and TLG could potentially have clinical value in evaluation of tumor biology, treatment response, and prognostication in cancer $(13,14)$. Recently, SUVmax and MTV are useful for differentiation of malignant from benign thyroid incidentaloma after stratification of serum thyroid stimulating hormone (TSH) levels (15). However, criteria for these volumetric parameters have not been established. In particular, the question of which is the better parameter to differentiate diagnosis of malignant and benign focal ${ }^{18} \mathrm{~F}-\mathrm{FDG}$ incidentaloma is unresolved. Kim and Chang (16) have also examined the clinical value of intratumoral heterogeneity of ${ }^{18} \mathrm{~F}$-FDG uptake represented as the heterogeneity factor for characterization of thyroid nodule with inconclusive fine-needle aspiration biopsy (FNAB) results. Objectives of this study were to compare semi-quantitative parameters of ${ }^{18} \mathrm{~F}-\mathrm{FDG} \mathrm{PET} / \mathrm{CT}$ for focal thyroid ${ }^{18} \mathrm{~F}-\mathrm{FDG}$ incidentaloma and assess clinical value of ${ }^{18} \mathrm{~F}-\mathrm{FDG} \mathrm{PET} / \mathrm{CT}$ for differentiation of malignant from benign focal thyroid ${ }^{18} \mathrm{~F}-\mathrm{FDG}$ incidentaloma.

\section{MATERIALS AND METHODS}

A total of 5216 individuals either healthy or had known or suspected cancer, attended Shanghai 85 Hospital September 2011-December 2014, enrolled in this study. Thyroid incidentalomas defined as unexpected focal hypermetabolic thyroid lesions identified by ${ }^{18} \mathrm{~F}-\mathrm{FDG}$ PET/CT. Thyroid incidentaloma patients with cytologically or histologically diagnosed by ultrasound and FNAB or surgery were included this study. Patients were excluded based on the following: 1) diffusely increased ${ }^{18} \mathrm{~F}$-FDG uptake throughout the thyroid gland; 2) with known thyroid cancer or a previous history of thyroid surgery; 3 ) no further evaluation on thyroid lesion due to patients' general condition; 4) follow-up loss; and 5) inconclusive results by ultrasound and FNAB. A total of 99 subjects ( 29 men and 70 women; mean age, 49.7 \pm 13.2 ; age range, 20-87) with hypermetabolic thyroid incidentalomas on FDG PET/CT were included in this study. We retrospectively reviewed medical records. Patients were cytologically or histologically diagnosed by ultrasound and FNAB or surgery.

\section{${ }^{18} \mathrm{~F}$-FDG PET/CT Imaging}

Fluorine-18-FDG PET/CT images were obtained using a dedicated PET/CT scanner (Discovery STE; GE Healthcare, Knoxville, TN, USA). Standard patient preparation included a fasting period of at least 6 hours and a serum glucose level lower than $6.5 \mathrm{mmol} / \mathrm{L}$ before ${ }^{18} \mathrm{~F}-\mathrm{FDG}$ administration. $\mathrm{PET} / \mathrm{CT}$ imaging was conducted 60 minutes after injection of ${ }^{18} \mathrm{~F}-\mathrm{FDG}$ ( $5 \mathrm{MBq} / \mathrm{kg}$ of body weight). CT scanning was conducted in a spiral mode from the skull base to the proximal thigh at $100 \mathrm{mAs}$ and $120 \mathrm{kV}$, with a section width of $5 \mathrm{~mm}$ and collimation of $0.75 \mathrm{~mm}$. CT scanning data were obtained for attenuation correction and image fusion and were followed by a three-dimensional craniocaudal PET emission scan. Whole body imaging was conducted from the base of the skull to the middle thigh with the arms up. Emission scan time per bed position was 3 minutes and six bed positions were acquired. PET data were obtained using a high-resolution whole-body scanner with an axial field of view of $21.6 \mathrm{~cm}$. After scatter and decay correction, PET data were reconstructed iteratively with attenuation correction and reoriented in axial, sagittal, and coronal slices. Average total PET/CT examination time was 20 minutes.

\section{${ }^{18} \mathrm{~F}$-FDG PET/CT Image Analysis}

Fluorodeoxyglucose PET/CT images were independently reviewed by two experienced nuclear physicians blinded to imaging studies and to clinical and pathologic results. Disagreements were resolved by consensus. Data were transferred via the Digital Imaging and Communications in Medicine protocol to a processing workstation (AW 4.4 workstation; General Electric Medical Systems, Knoxville, TN, USA).

Standardized uptake values were determined by regionof-interest (ROI) technique. To calculate SUVmax and SUVmean, manually defined circular ROI were drawn on 
attenuation-corrected emission images throughout axial planes in which a suspicious lesion could be delineated. An iso-contour connecting outlines of the volume of interest (VOI) was set using different cutoff values for SUV (i.e., 2.5, 3.0, 3.5, and 4.0). The contour around the target lesion inside the boundaries was automatically produced, equal to or greater than each threshold of SUV of the primary tumor on each axial image of PET/CT. After hypermetabolic tumor foci were segmented, MTV was calculated by summation of voxels of each slice of PET/CT (Fig. 1).

Total lesion glycolysis was calculated by multiplying selected PET volume by average SUV within that volume:

$$
T L G=M T V \times(\text { average SUV) }
$$

\section{Statistical Analysis}

Continuous variables are expressed as means \pm standard

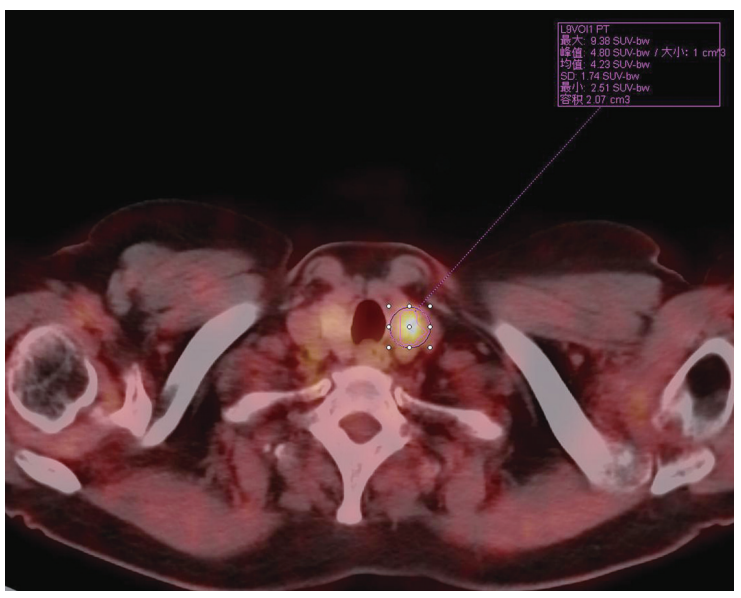

A

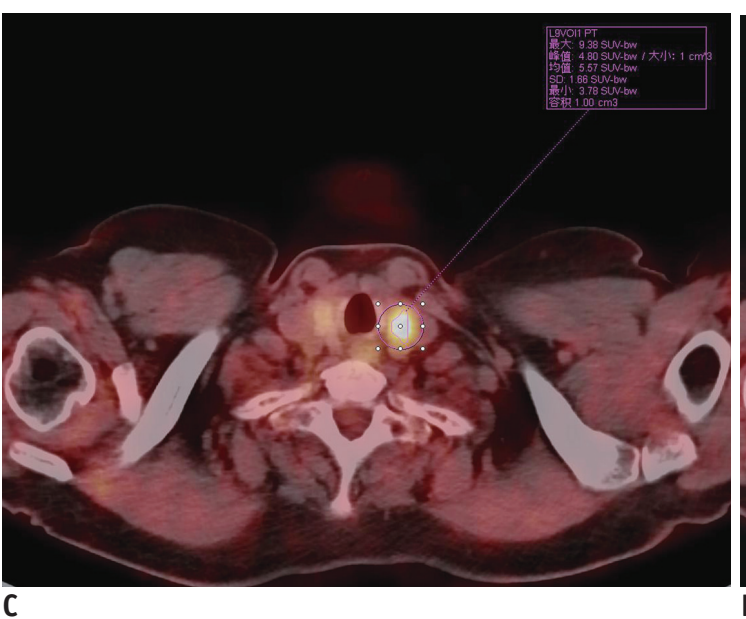

deviations, medians and ranges, and categorical variables as numbers and percentages. Clinical and demographic characteristics were compared using $\chi^{2}$ test or Fisher's exact test. Thyroid ${ }^{18} \mathrm{~F}$-FDG incidentaloma size, SUVmax, MTV and TLG in benign and malignant lesions were compared using the Mann-Whitney $U$ test. Receiver-operating characteristic (ROC) analysis was conducted to evaluate usefulness of SUVmax, MTV and TLG, as markers for differentiating malignant from benign thyroid incidentalomas. The area under the ROC curve (areas under the curve $[A U C]$ ) was calculated and ranged from $0.5-1.0$, increasing when diagnostic performance approached reference standard (in this study, determination of malignancy) and AUC of semiquantitative parameters assessed by ${ }^{18} \mathrm{~F}$-FDG PET/CT were compared by Hanley and McNeil test. Feasible threshold of FDG PET/CT images parameters were tested for capability to differentiate between malignant and benign thyroid
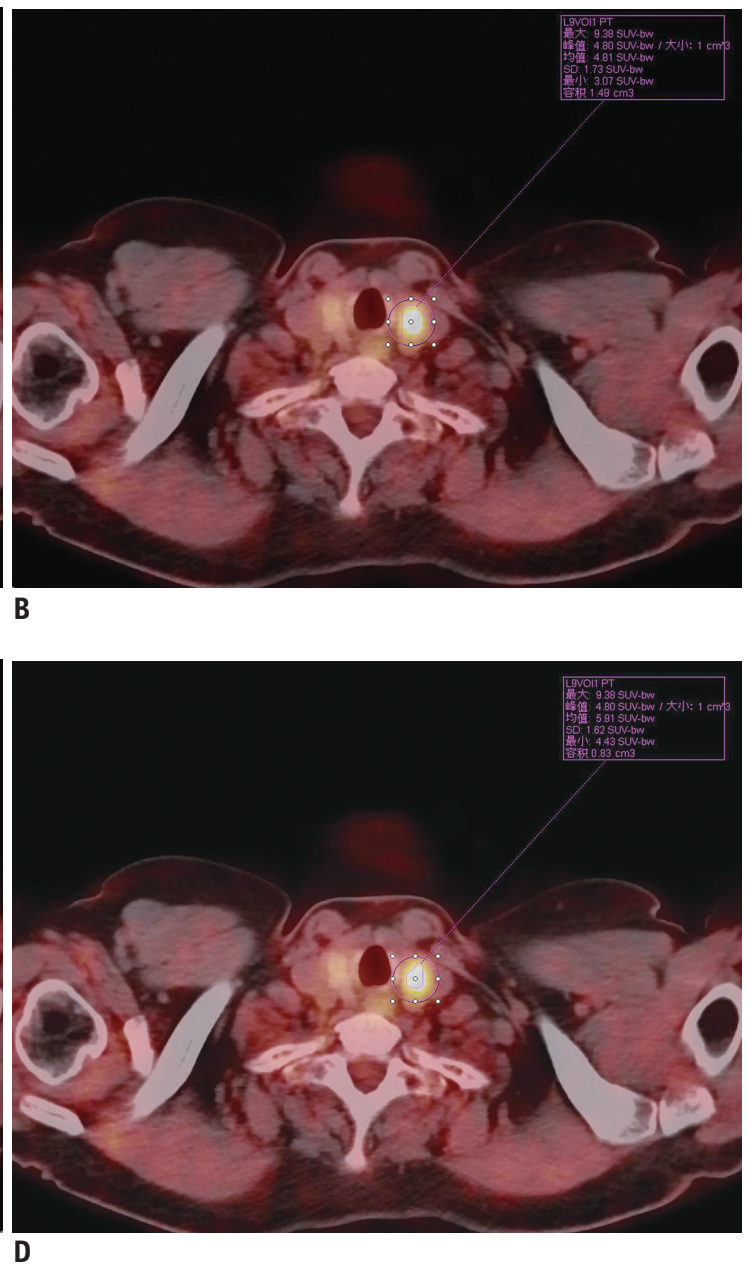

Fig. 1. Illustration of method of measurement of MTV 2.5 (A), MTV 3.0 (B), MTV 3.5 (C), and MTV 4.0 (D) of thyroid ${ }^{18} \mathrm{~F}-\mathrm{FDG}$ incidentaloma by ${ }^{18}$ F-FDG PET/CT. MTV 2.5, MTV 3.0, MTV 3.5, and MTV 4.0 were 2.07, 1.49, 1.00 , and $0.83 \mathrm{~cm}^{3}$, respectively. MTV = metabolic tumor volume, $\mathrm{PET} / \mathrm{CT}=$ positron emission tomography/computed tomography, SUV = standardized uptake value, ${ }^{18} \mathrm{~F}-\mathrm{FDG}=\mathrm{fluorine}-18-$ fluorodeoxyglucose 
incidentalomas.

All $p$ values reported were two-tailed, and a $p$ value of less than 0.05 was statistically significant. All statistical analysis was conducted with SPSS software (version 10.0; SPSS Inc., Chicago, IL, USA).

\section{RESULTS}

\section{Patients' Clinical Characteristics}

Mean diameter of thyroid incidentaloma was $1.6 \pm 0.8 \mathrm{~cm}$ (range, $0.5-4.2 \mathrm{~cm}$ ). In all 99 incidentalomas, a histological

Table 1. Histological Diagnoses of 99 Thyroid Incidentalomas

\begin{tabular}{lc}
\hline \multicolumn{1}{c}{ Lesion Type and Diagnosis } & No. of Lesions* \\
\hline Malignant & $64(64.6 \%)$ \\
\hline Papillary adenocarcinoma & 38 \\
Medullary carcinoma & 8 \\
\hline Thyroid follicular adenocarcinoma & 10 \\
Anaplastic thyroid carcinoma & 7 \\
Metastatic carcinoma & 1 \\
Benign & $35(35.4 \%)$ \\
Thyroid adenoma & 29 \\
Colloid retention cyst & 1 \\
\hline Nodular hyperplasia & 1 \\
\hline Nodular goiter & 2 \\
\hline Hashimoto's thyroiditis & 2 \\
\hline
\end{tabular}

*Data in parentheses are percentages. reference diagnosis was made by surgical resection $(\mathrm{n}=$ $52)$ or ultrasound and FNAB $(n=47)$. Of the 99 thyroid incidentalomas, 64 (64.6\%) were malignant and 35 (35.4\%) were benign (Table 1). Papillary adenocarcinoma was the main pathological type of malignant thyroid incidentaloma (38/64, 59.4\%); and thyroid adenoma was common in benign thyroid incidentaloma (29/35, 82.9\%).

Comparisons of patient age, sex, and diameter between malignant and benign thyroid incidentalomas are summarized in Table 2. There were no statistically significant differences between malignant and benign thyroid incidentalomas for patient age ( $48.8 \pm 14.7$ vs. 51.2 $\pm 9.8, p=0.370)$ and sex (20/44 vs. $9 / 26, p=0.563)$.

Malignant thyroid incidentalomas were larger than benign lesions ( $1.8 \pm 0.8 \mathrm{~cm}$ vs. $1.3 \pm 0.5 \mathrm{~cm}, p=0.006)$. Results of ROC curve analysis revealed that a threshold diameter of $1.2 \mathrm{~cm}$ had $79.7 \%$ sensitivity and $48.6 \%$ specificity for identifying malignant thyroid incidentalomas with AUC of 0.667 (95\% confidence interval [CI]: 0.556-0.777).

Maximum standardized uptake value of malignant and benign thyroid incidentalomas.

Comparisons of SUVmax of malignant and benign thyroid incidentalomas are summarized in Table 3. There were statistically significant differences between malignant and benign thyroid incidentalomas for SUVmax $(11.3 \pm 8.4$ vs. $4.8 \pm 5.3, p<0.001)$. Malignant thyroid incidentalomas

Table 2. Comparisons of Patient Characteristics for Malignant and Benign Thyroid Incidentalomas

\begin{tabular}{lccc}
\hline & Malignant & Benign & $P^{\star}$ \\
\hline Age (years, mean \pm SD) & $48.8 \pm 14.7$ & $51.2 \pm 9.8$ & 0.370 \\
Sex (male/female) & $20 / 44$ & $9 / 26$ & 0.563 \\
Diameter $(c m$, mean \pm SD) & $1.8 \pm 0.8$ & $1.3 \pm 0.5$ & 0.006 \\
\hline
\end{tabular}

*Mann-Whitney $U$ test performed to analyze statistically significant differences in patient age and diameter of malignant and benign nodules. $\chi^{2}$ test used to analyze statistically significant differences in patient gender. SD = standard deviation

Table 3. Malignant and Benign Thyroid Incidentalomas Characteristics at ${ }^{18} \mathrm{~F}-\mathrm{FDG}$ PET/CT

\begin{tabular}{lcccccccc}
\hline & Mean \pm SD & $\begin{array}{c}\text { Malignant } \\
\text { Median }\end{array}$ & Range & Mean \pm SD & $\begin{array}{c}\text { Benign } \\
\text { Median }\end{array}$ & Range & $P^{*}$ & AUC (95\% CI) \\
\hline SUVmax & $11.3 \pm 8.4$ & 9.800 & $2.10,47.00$ & $4.8 \pm 5.3$ & 3.60 & $1.40,26.90$ & $<0.001$ & $0.866(0.783,0.950)$ \\
MTV 2.5 $\left(\mathrm{cm}^{3}\right)$ & $3.3 \pm 5.2$ & 1.370 & $0.10,26.40$ & $0.8 \pm 0.9$ & 0.50 & $0.04,3.60$ & $<0.001$ & $0.764(0.667,0.861)$ \\
MTV 3.0 $\left(\mathrm{cm}^{3}\right)$ & $3.0 \pm 4.7$ & 1.245 & $0.07,22.80$ & $0.6 \pm 0.7$ & 0.34 & $0.03,2.70$ & $<0.001$ & $0.796(0.707,0.886)$ \\
MTV 3.5 $\left(\mathrm{cm}^{3}\right)$ & $2.8 \pm 4.2$ & 1.215 & $0.05,20.10$ & $0.5 \pm 0.6$ & 0.22 & $0.01,2.35$ & $<0.001$ & $0.819(0.735,0.903)$ \\
MTV 4.0 (cm $\left.{ }^{3}\right)$ & $2.7 \pm 4.0$ & 1.185 & $0.04,19.20$ & $0.3 \pm 0.5$ & 0.13 & $0.01,2.31$ & $<0.001$ & $0.872(0.802,0.942)$ \\
TLG 2.5 & $37.2 \pm 92.8$ & 10.140 & $0.16,644.00$ & $3.5 \pm 7.1$ & 0.84 & $0.05,37.75$ & $<0.001$ & $0.827(0.743,0.911)$ \\
TLG 3.0 & $34.2 \pm 86.1$ & 9.270 & $0.13,607.60$ & $2.8 \pm 6.6$ & 0.74 & $0.03,37.28$ & $<0.001$ & $0.851(0.772,0.930)$ \\
TLG 3.5 & $31.7 \pm 79.7$ & 8.790 & $0.10,562.80$ & $2.5 \pm 6.4$ & 0.53 & $0.01,36.66$ & $<0.001$ & $0.867(0.791,0.942)$ \\
TLG 4.0 & $30.0 \pm 75.5$ & 8.635 & $0.07,537.60$ & $1.9 \pm 6.2$ & 0.27 & $0.01,36.03$ & $<0.001$ & $0.895(0.826,0.963)$ \\
\hline
\end{tabular}

*Mann-Whitney $\mathrm{U}$ test. AUC = areas under the curve, CI = confidence interval, MTV = metabolic tumor volume, SUVmax = maximum standardized uptake value, TLG = total lesion glycolysis, ${ }^{18} \mathrm{~F}-\mathrm{FDG} \mathrm{PET} / \mathrm{CT}$ = fluorine-18-fluorodeoxyglucose positron emission tomography/ computed tomography 
had higher SUVmax than benign. Results of ROC curve analysis (Fig. 2) revealed that a threshold SUVmax of 4.45 had $90.6 \%$ sensitivity and $68.6 \%$ specificity for identifying malignant thyroid incidentalomas with AUC of 0.866 (95\% CI: 0.783-0.950).

\section{MTV of Malignant and Benign Thyroid Incidentalomas}

Comparisons of MTV of malignant and benign thyroid incidentalomas are summarized in Table 3. All four different PET volumes tested (MTV 2.5, MTV 3.0, MTV 3.5, and MTV 4.0) of malignant thyroid incidentalomas were larger than those of benign lesions (all $p<0.001$ ). Results of ROC curve analysis (Fig. 3 ) revealed that AUC of MTV 2.5, MTV 3.0, MTV 3.5, and MTV 4.0 were $0.764(p<0.001), 0.796$ $(p<0.001), 0.819(p<0.001)$, and $0.872(p<0.001)$, respectively. And MTV 4.0 had highest performance of differentiation of malignant from benign thyroid ${ }^{18} \mathrm{~F}-\mathrm{FDG}$ incidentaloma in all MTV indexes with AUC 0.872 by ROC curve analysis. A threshold MTV 4.0 of $0.325 \mathrm{~cm}^{3}$ had $85.9 \%$ sensitivity and $71.4 \%$ specificity for identifying malignant thyroid incidentalomas.

\section{TLG of Malignant and Benign Thyroid Incidentalomas}

Comparisons of TLG of malignant and benign thyroid incidentalomas are summarized in Table 3. All four different TLGs calculated (TLG 2.5, TLG 3.0, TLG 3.5, and TLG 4.0) of

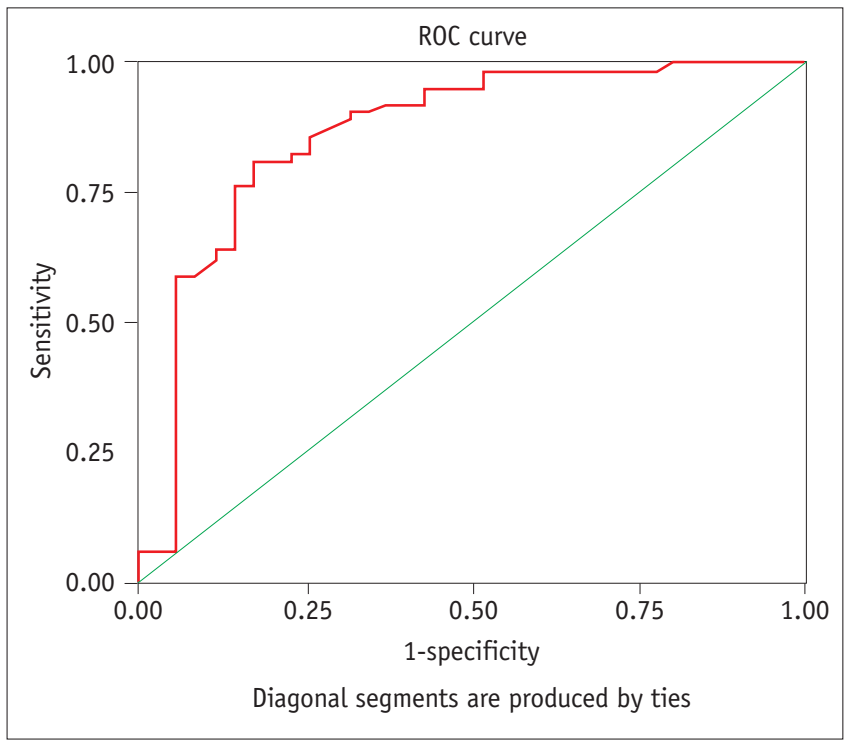

Fig. 2. Graph shows results of ROC analysis for differentiating malignant from benign thyroid incidentalomas by SUVmax. AUC was 0.866 . Threshold SUVmax of 4.45 had $90.6 \%$ sensitivity and $68.6 \%$ specificity for identifying malignant thyroid incidentalomas. $A U C=$ areas under the curve, $\mathrm{ROC}=$ receiver-operator characteristic, SUVmax $=$ maximum standardized uptake value malignant thyroid incidentalomas were larger than those of benign lesions (all $p<0.001$ ). Results of ROC curve analysis (Fig. 4) revealed that AUC of TLG 2.5, TLG 3.0, TLG 3.5, and TLG 4.0 were $0.827(p<0.001), 0.851(p<0.001), 0.867(p$ $<0.001)$, and $0.895(p<0.001)$, respectively. TLG 4.0 had highest performance of differentiation of malignant from benign thyroid ${ }^{18} \mathrm{~F}-\mathrm{FDG}$ incidentaloma in all TLG indexes with AUC of 0.895 by ROC curve analysis. A threshold TLG 4.0 of 2.475 had $81.3 \%$ sensitivity and $94.3 \%$ specificity for identifying malignant thyroid incidentalomas.

\section{Comparisons of AUCs of the PET/CT Parameters}

Areas under the curve of TLG 4.0 was significantly larger than those of diameter, SUVmean, MTV 2.5, MTV 3.0, MTV 3.5, TLG 2.5, and TLG 3.0, respectively (all $p<0.05$ ). But there was no significant statistical difference between AUC of TLG 4.0 and AUC of SUVmax, AUC of MTV 4.0, or AUC of TLG 3.5 (all $p>0.05$ ).

\section{Characteristic of Primary Malignant Thyroid Incidentalomas on ${ }^{18} \mathrm{~F}-\mathrm{FDG}$ PET/CT}

Comparisons of semi-quantitative parameters assessed by ${ }^{18} \mathrm{~F}-\mathrm{FDG} \mathrm{PET} / \mathrm{CT}$ characteristic of primary malignant thyroid incidentalomas are summarized in Table 4. Of the 99 thyroid incidentalomas, $63(63.6 \%)$ were primary malignant thyroid tumor. of 63 primary malignant thyroid tumors,

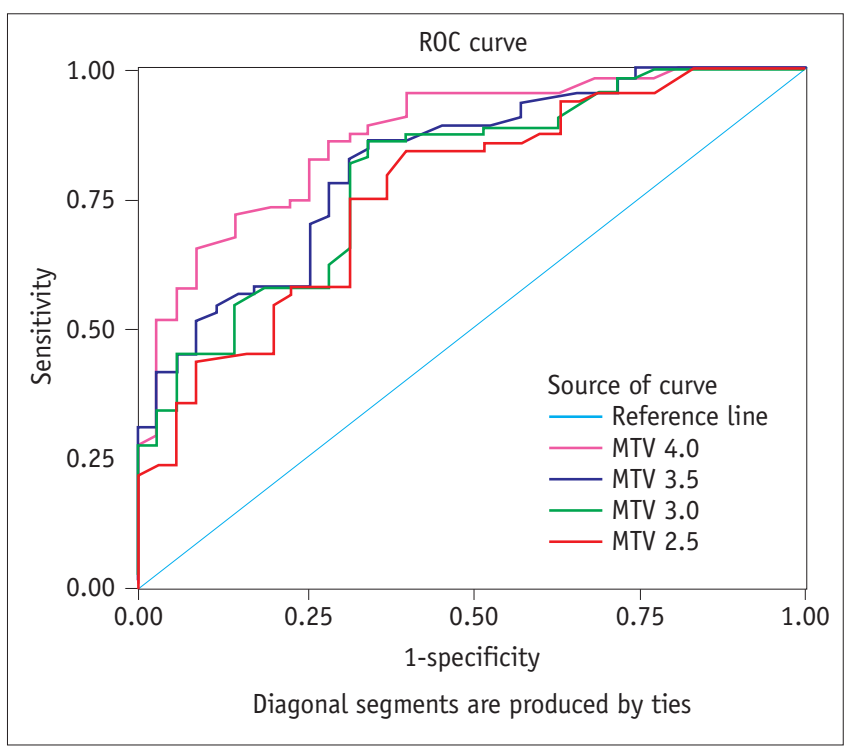

Fig. 3. Graph shows results of ROC analysis for differentiating malignant from benign thyroid incidentalomas by MTV 2.5, MTV 3.0, MTV 3.5, and MTV 4.0. AUC were 0.764, 0.796, 0.819, and 0.872 , respectively. Threshold MTV 4.0 of $0.325 \mathrm{~cm}^{3}$ had $85.9 \%$ sensitivity and $71.4 \%$ specificity for identifying malignant thyroid incidentalomas. 


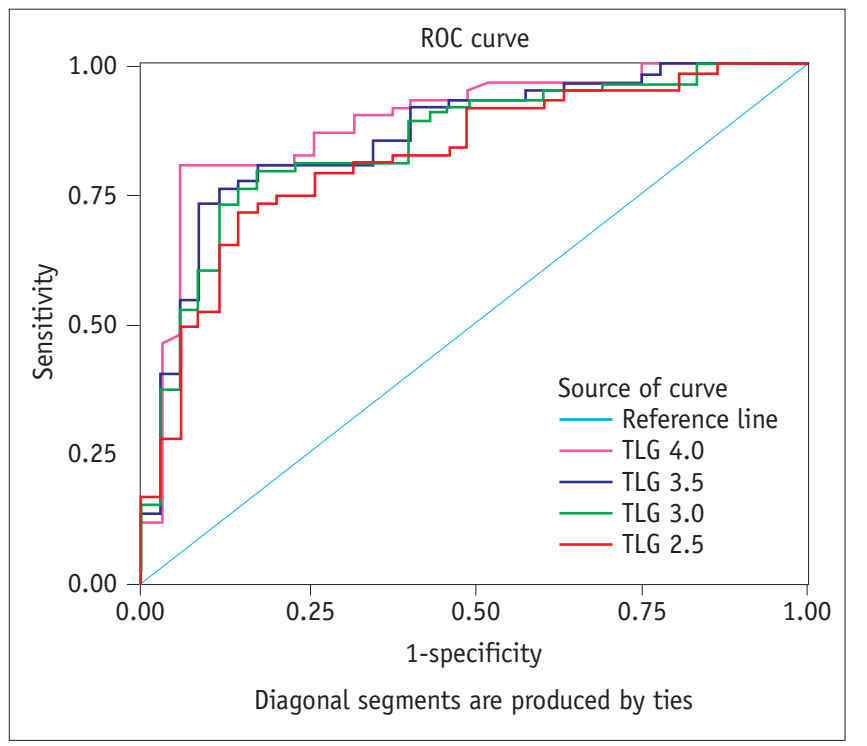

Fig. 4. Graph shows results of ROC analysis for differentiating malignant and benign thyroid incidentalomas by TLG 2.5, TLG 3.0, TLG 3.5, and TLG 4.0. AUC were 0.827, 0.851, 0.867, and 0.895, respectively. Threshold TLG 4.0 of 2.475 had $81.3 \%$ sensitivity and $94.3 \%$ specificity for identifying malignant thyroid incidentalomas. TLG $=$ total lesion glycolysis

the most common primary malignant thyroid tumor was papillary adenocarcinoma $(60.3 \%, 38 / 63) ; 10(15.9 \%)$ were thyroid follicular adenocarcinomas; 8 (12.7\%) were thyroid medullary carcinomas; other $7(11.1 \%)$ were anaplastic thyroid carcinomas. There were no statistically significant differences of semi-quantitative ${ }^{18} \mathrm{~F}$-FDG PET parameters within different primary malignant thyroid incidentalomas (all $p>0.05$ ).

\section{DISCUSSION}

This study investigated diagnostic values of semiquantitative parameters assessed by ${ }^{18} \mathrm{~F}-\mathrm{FDG}$ PET/CT for differentiation of malignant from benign thyroid ${ }^{18} \mathrm{~F}-\mathrm{FDG}$ incidentaloma. Results revealed that various semi-quantitative parameters (i.e., SUVmax, MTV, and TLG) assessed by ${ }^{18} \mathrm{~F}-\mathrm{FDG}$ PET/CT were different between malignant and benign thyroid ${ }^{18} \mathrm{~F}-\mathrm{FDG}$ incidentaloma, that could contribute to differentiation of malignant from benign thyroid ${ }^{18} \mathrm{~F}$-FDG incidentaloma. Our study also revealed that semi-quantitative parameters (i.e., SUVmax, MTV, and TLG) were not significantly different within pathological subtypes of primary malignant thyroid ${ }^{18} \mathrm{~F}-\mathrm{FDG}$ incidentaloma.

Thyroid incidentalomas on FDG PET/CT are currently a subject of major interest because of emerging practical use of FDG PET/CT in cancer evaluation. Prevalence of thyroid incidentaloma on FDG PET/CT is reported to be approximately $1.1-4.0 \%(8,9,11,17-19)$. According to recent meta-analysis investigating 147505 units, the pooled incidence of thyroid incidentalomas detected by FDG PET/ CT was $2.46 \%$, malignancy ratio was $34.6 \%$ (20). In this study, thyroid incidentalomas on FDG PET/CT were identified with prevalence of $1.9 \%(99 / 5216)$ and the malignancy ratio was $64.6 \%$ (64/99). Prevalence of malignant thyroid incidentaloma was higher than reported in previous studies, which has been between 26.7 and $50 \%(8,9,11,17-20)$. This discordance may be related to excluding patients with diffuse thyroid FDG PET uptake in this study.

This considerably high prevalence of malignancy justifies further work-up, such as ultrasound and ultrasoundguided FNAB. Although FNAB is the most accurate and cost-effective method for evaluating thyroid nodules, accuracy of FNAB depends mainly on skill and experience of the investigator and cytopathologist. It is essential to differentiate malignant from benign thyroid ${ }^{18} \mathrm{~F}-\mathrm{FDG}$ incidentaloma by ${ }^{18} \mathrm{~F}-\mathrm{FDG}$ PET/CT because of wide use of FDG PET/CT in clinical practice. The role of FDG PET/ $\mathrm{CT}$ in the differentiating diagnosis of thyroid ${ }^{18} \mathrm{~F}-\mathrm{FDG}$ incidentaloma is under debate. Several studies reported higher SUVmax in malignant lesions than in benign ones $(5,19)$ and a positive correlation between presence of SUVmax $>5.0$ and diagnosis of malignancy (5). Ho et al. $(21,22)$ found a significantly higher average value of SUVmax in malignant thyroid lesions, but the authors were unable to establish an optimal SUVmax cutoff value to differentiate benign from malignant lesions, because there was considerable overlap in SUVmax between malignant and benign thyroid ${ }^{18} \mathrm{~F}-\mathrm{FDG}$ incidentalomas. This overlap makes it difficult to use SUVmax to differentiate malignant from benign thyroid ${ }^{18} \mathrm{~F}-\mathrm{FDG}$ incidentaloma. However, a number of studies revealed no significant difference in SUVmax between benign and malignant nodules $(9,17,21)$. Our study revealed malignant thyroid incidentalomas had higher SUVmax than benign. Results of ROC curve analysis revealed that a threshold SUVmax of 4.45 had $90.6 \%$ sensitivity and $68.6 \%$ specificity for identifying malignant thyroid incidentalomas.

Metabolic tumor volume and TLG are measurements of metabolic activity of tumors determined by ${ }^{18} \mathrm{~F}-\mathrm{FDG}$ PET/CT images (23). Unlike SUVmax, that reflects only the point of greatest metabolic activity within the tumor, volume based metabolic parameters of MTV could potentially have clinical value in evaluation of tumor biology, evaluation of response 


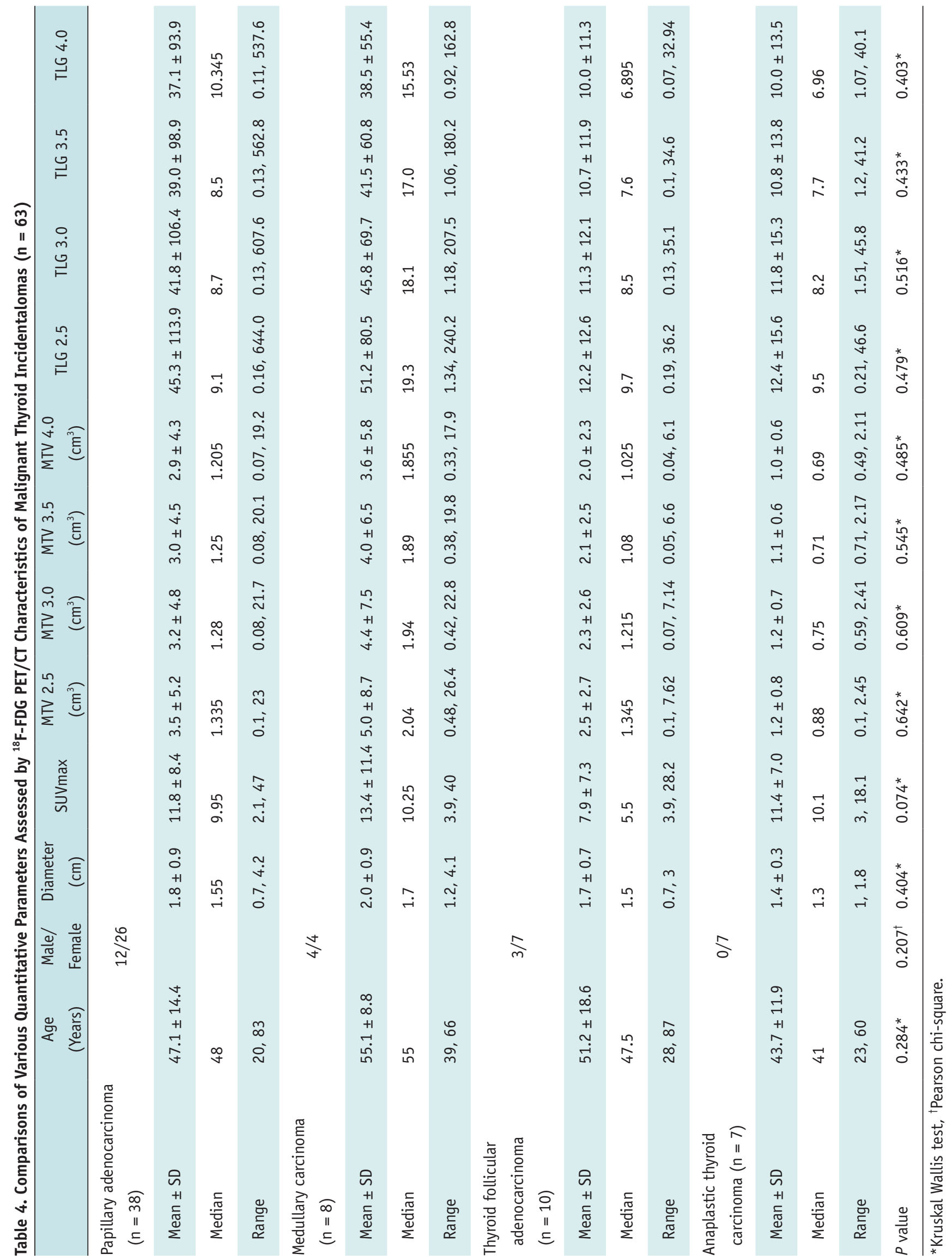


to treatment, and prognostication in various cancers (24). TLG, calculated by multiplying the SUV mean by the MTV (25), has been suggested to better reflect global metabolic activity in whole tumors (25). TLG has potential to become a valuable imaging biomarker in prognostic studies on human solid tumors, adding value to clinical staging, as well as in treatment response assessment and treatment optimization (24). However, few published studies have adapted these quantitative indexes for differentiating malignant from benign thyroid ${ }^{18} \mathrm{~F}-\mathrm{FDG}$ incidentaloma and the conclusion is under debate $(15,16)$. In the Kim et al. (15) study, patients with higher serum TSH levels (TSH > $1.31 \mathrm{mIU} / \mathrm{mL}$ ), malignant thyroid ${ }^{18} \mathrm{~F}-\mathrm{FDG}$ incidentaloma revealed statistically significantly higher MTV 4.0 compared with benign ones. However, MTV 3.5, MTV 3.0, and MTV 2.5 revealed no statistical differences between malignant and benign thyroid ${ }^{18} \mathrm{~F}-\mathrm{FDG}$ incidentaloma (15). In comparison ROC curve analysis, no significant difference was detected between SUVmax and MTV 4.0 in prediction of malignant thyroid ${ }^{18} \mathrm{~F}$-FDG incidentaloma. However, the combination of SUVmax and MTV 4.0 improved predictive value compared with individual AUCs of SUVmax and MTV 4.0 (15). Kim and Chang (16) reported no significant difference in MTV and TLG between malignant and benign thyroid ${ }^{18} \mathrm{~F}-\mathrm{FDG}$ incidentalomas. Our study revealed malignant thyroid ${ }^{18} \mathrm{~F}$-FDG incidentaloma had statistically significantly higher MTV indexes (including MTV 4.0, MTV 3.5, MTV 3.0, and MTV 2.5) and TLG indexes (including TLG 4.0, TLG 3.5, TLG 3.0, and TLG 2.5) compared with benign ones. In comparison ROC curve analysis, MTV 4.0 has highest performance $(85.9 \%$ sensitivity and $71.4 \%$ specificity, $A \cup C=0.872)$ of differentiating malignant from benign thyroid ${ }^{18} \mathrm{~F}-\mathrm{FDG}$ incidentaloma in all MTV indexes, TLG 4.0 has highest performance $(81.3 \%$ sensitivity and $94.3 \%$ specificity, $A U C=0.895)$ in all TLG indexes. TLG 4.0 revealed highest performance for differentiating malignant from benign thyroid ${ }^{18} \mathrm{~F}$-FDG incidentaloma with highest AUC in all semiquantitative parameters assessed by ${ }^{18} \mathrm{~F}-\mathrm{FDG}$ PET/CT. AUC (TLG 4.0) were significantly larger than AUC (SUVmean), AUC (MTV 2.5), AUC (MTV 3.0), AUC (MTV 3.5), AUC (TLG 2.5) and AUC (TLG 3.0), respectively. There were no significant statistical difference between AUC (TLG 4.0) and AUC (SUVmax), AUC (MTV 4.0), or AUC (TLG 3.5). Results from Kim and Chang's study are discordant with our study (16). This discordance may be related to composition of the study sample. Malignancy ratio was $64.6 \%$ (64/99) in our study sample, but only $24.5 \%$ (49/200) in Kim and Chang's study sample (16). Their study also revealed malignant incidental thyroid lesions had higher ${ }^{18} \mathrm{~F}$-FDG PET/CT parameters including SUVmax, MTV, and TLG than benign, but there was no statistical difference.

This retrospective single center study has limited sample size of thyroid incidentaloma patients. We did not include diffuse thyroid FDG PET uptake because previous reports indicate that the majority represent chronic thyroiditis or diffuse thyroid autonomy and do not need histological diagnosis, except for a small number of conditions (26). Additional, VOI selection is essential for determining tumor MTV. Several different methods for VOI selection could be used: manually defined VOI, iso-contour VOIs based on a fixed percentage of the SUVmax, and iso-contour VOIs based on a fixed SUVmax threshold. This study used the fixed SUVmax threshold method, that may be subject to issues arising from SUV variability. Therefore, to minimize issues, we used various thresholds of SUVmax for VOI selection.

Conventional quantitative PET parameters only derived from ${ }^{18} \mathrm{~F}$-FDG PET/CT were not adequate to differentiate benign from malignant thyroid incidentalomas. To overcome this, the Hounsfield unit ratio of thyroid nodules was assessed compared to the contralateral thyroid lobe on noncontrast CT to stratify further risk of malignancy in thyroid incidentalomas detected in ${ }^{18} \mathrm{~F}$-FDG PET/CT (27). Dual-timepoint ${ }^{18} \mathrm{~F}-\mathrm{FDG} \mathrm{PET} / \mathrm{CT}$ and retention index were used in differential diagnosis of thyroid incidentaloma (28). Barrio et al. (29) measured SUVmax, thyroid to background thyroid lesion SUVmax/thyroid background SUVmean (TL/TBG), thyroid to blood pool TL/blood pool SUVmean (BP), and thyroid to liver TL/liver SUVmean $(\mathrm{L})$ ratios in benign and malignant lesions to test if intense focal ${ }^{18} \mathrm{~F}-\mathrm{FDG}$ thyroid uptake is associated with malignancy. These studies revealed various methods may significantly improve accuracy of PET/ CT for differentiating benign from malignant focal thyroid lesions.

In conclusion, this study has revealed that volume-based $\mathrm{PET} / \mathrm{CT}$ parameters such as TLG and MTV combining tumor volume and metabolic activity of the entire tumor, could potentially have clinical value in differential diagnosis of thyroid incidentaloma along with SUVmax. Considering that the measurement of volume-based PET parameters required additional time and effort to the measurement of SUVmax, the clinical value of volume-based PET functional parameters in differential diagnosis of thyroid ${ }^{18} \mathrm{~F}-\mathrm{FDG}$ incidentaloma should be further determined in future prospective studies with a large sample size, and combined 
CT pattern analysis are also needed.

\section{REFERENCES}

1. Burguera B, Gharib H. Thyroid incidentalomas. Prevalence, diagnosis, significance, and management. Endocrinol Metab Clin North Am 2000;29:187-203

2. Poeppel TD, Krause BJ, Heusner TA, Boy C, Bockisch A, Antoch G. PET/CT for the staging and follow-up of patients with malignancies. Eur J Radiol 2009;70:382-392

3. Bomanji JB, Costa DC, Ell PJ. Clinical role of positron emission tomography in oncology. Lancet Oncol 2001;2:157164

4. Chen YK, Ding HJ, Chen KT, Chen YL, Liao AC, Shen YY, et al. Prevalence and risk of cancer of focal thyroid incidentaloma identified by $18 \mathrm{~F}$-fluorodeoxyglucose positron emission tomography for cancer screening in healthy subjects. Anticancer Res 2005;25:1421-1426

5. Pagano L, Samà MT, Morani F, Prodam F, Rudoni M, Boldorini $R$, et al. Thyroid incidentaloma identified by ${ }^{18} \mathrm{~F}$-fluorodeoxyglucose positron emission tomography with CT (FDG-PET/CT): clinical and pathological relevance. Clin Endocrinol (Oxf) 2011;75:528-534

6. Karantanis D, Bogsrud TV, Wiseman GA, Mullan BP, Subramaniam RM, Nathan MA, et al. Clinical significance of diffusely increased $18 \mathrm{~F}-\mathrm{FDG}$ uptake in the thyroid gland. $\mathrm{J}$ Nucl Med 2007;48:896-901

7. Liu Y. Clinical significance of thyroid uptake on F18fluorodeoxyglucose positron emission tomography. Ann NuCl Med 2009;23:17-23

8. Are C, Hsu JF, Schoder H, Shah JP, Larson SM, Shaha AR. FDG-PET detected thyroid incidentalomas: need for further investigation? Ann Surg Oncol 2007;14:239-247

9. Kim TY, Kim WB, Ryu JS, Gong G, Hong SJ, Shong YK. 18F-fluorodeoxyglucose uptake in thyroid from positron emission tomogram (PET) for evaluation in cancer patients: high prevalence of malignancy in thyroid PET incidentaloma. Laryngoscope 2005;115:1074-1078

10. Kim BH, Na MA, Kim IJ, Kim SJ, Kim YK. Risk stratification and prediction of cancer of focal thyroid fluorodeoxyglucose uptake during cancer evaluation. Ann Nucl Med 2010;24:721728

11. Kang KW, Kim SK, Kang HS, Lee ES, Sim JS, Lee IG, et al. Prevalence and risk of cancer of focal thyroid incidentaloma identified by $18 \mathrm{~F}$-fluorodeoxyglucose positron emission tomography for metastasis evaluation and cancer screening in healthy subjects. J Clin Endocrinol Metab 2003;88:4100-4104

12. Bogsrud TV, Karantanis D, Nathan MA, Mullan BP, Wiseman GA, Collins DA, et al. The value of quantifying $18 \mathrm{~F}-\mathrm{FDG}$ uptake in thyroid nodules found incidentally on whole-body PET-CT. Nucl Med Commun 2007;28:373-381

13. Dibble EH, Alvarez AC, Truong MT, Mercier G, Cook EF, Subramaniam RM. 18F-FDG metabolic tumor volume and total glycolytic activity of oral cavity and oropharyngeal squamous cell cancer: adding value to clinical staging. J Nucl Med 2012;53:709-715

14. I HS, Kim SJ, Kim IJ, Kim K. Predictive value of metabolic tumor volume measured by 18F-FDG PET for regional lymph node status in patients with esophageal cancer. Clin Nucl Med 2012;37:442-446

15. Kim BH, Kim SJ, Kim H, Jeon YK, Kim SS, Kim IJ, et al. Diagnostic value of metabolic tumor volume assessed by 18F-FDG PET/CT added to SUVmax for characterization of thyroid 18F-FDG incidentaloma. Nucl Med Commun 2013;34:868-876

16. Kim SJ, Chang S. Predictive value of intratumoral heterogeneity of F-18 FDG uptake for characterization of thyroid nodules according to Bethesda categories of fine needle aspiration biopsy results. Endocrine 2015;50:681-688

17. Nam SY, Roh JL, Kim JS, Lee JH, Choi SH, Kim SY. Focal uptake of (18)F-fluorodeoxyglucose by thyroid in patients with nonthyroidal head and neck cancers. Clin Endocrinol (Oxf) 2007;67:135-139

18. Cohen MS, Arslan N, Dehdashti F, Doherty GM, Lairmore TC, Brunt LM, et al. Risk of malignancy in thyroid incidentalomas identified by fluorodeoxyglucose-positron emission tomography. Surgery 2001;130:941-946

19. Makis W, Ciarallo A. Thyroid incidentalomas on 18F-FDG PET/ CT: clinical significance and controversies. Mol Imaging Radionucl Ther 2017;26:93-100

20. Choi JY, Lee KS, Kim HJ, Shim YM, Kwon 0J, Park K, et al. Focal thyroid lesions incidentally identified by integrated 18F-FDG PET/CT: clinical significance and improved characterization. J Nucl Med 2006;47:609-615

21. Ho TY, Liou MJ, Lin KJ, Yen TC. Prevalence and significance of thyroid uptake detected by ${ }^{18} \mathrm{~F}-\mathrm{FDG}$ PET. Endocrine 2011;40:297-302

22. Bertagna F, Treglia G, Piccardo A, Giubbini R. Diagnostic and clinical significance of F-18-FDG-PET/CT thyroid incidentalomas. J Clin Endocrinol Metab 2012;97:3866-3875

23. Kim H, Kim SJ, Kim IJ, Kim K. Thyroid incidentalomas on FDG PET/CT in patients with non-thyroid cancer - a large retrospective monocentric study. Onkologie 2013;36:260-264

24. Erdi YE, Macapinlac H, Rosenzweig KE, Humm JL, Larson SM, Erdi AK, et al. Use of PET to monitor the response of lung cancer to radiation treatment. Eur J Nucl Med 2000;27:861866

25. Van de Wiele C, Kruse V, Smeets P, Sathekge M, Maes A. Predictive and prognostic value of metabolic tumour volume and total lesion glycolysis in solid tumours. Eur J Nucl Med Mol Imaging 2013;40:290-301

26. Salvatori M, Melis L, Castaldi P, Maussier ML, Rufini V, Perotti $G$, et al. Clinical significance of focal and diffuse thyroid diseases identified by (18)F-fluorodeoxyglucose positron emission tomography. Biomed Pharmacother 2007;61:488-493

27. Kim D, Hwang SH, Cha J, Jo K, Lee N, Yun M. Risk stratification of thyroid incidentalomas found on PET/ CT: the value of iodine content on noncontrast computed tomography. Thyroid 2015;25:1249-1254 
28. Lee S, Park T, Park S, Pahk K, Rhee S, Cho J, et al. The clinical role of dual-time-point (18)F-FDG PET/CT in differential diagnosis of the thyroid incidentaloma. Nucl Med Mol Imaging 2014;48:121-129

29. Barrio M, Czernin J, Yeh MW, Palma Diaz MF, Gupta P, Allen-
Auerbach $M$, et al. The incidence of thyroid cancer in focal hypermetabolic thyroid lesions: an ${ }^{18} \mathrm{~F}-\mathrm{FDG}$ PET/CT study in more than 6000 patients. Nucl Med Commun 2016;37:12901296 